Also, the fact that the inhibitor does not appear to be an aromatic compound conflicts with the suggested nature of inhibitor $\beta$ in several other species of plants and plant organs. Varga ${ }^{4}$ has extracted growth-inhibitors from several species of fleshy fruits and concludes that inhibitor $\beta$ is composed of several aromatic compounds and, in particular, phenolic compounds. In Citrus medica L., Varga has identified three phenolic compounds which are present in eluates of inhibitor $\beta$, namely, salicylic acid, $o$-coumaric acid and either $p$-coumaric acid or ferulic acid. Ether extracts of $C$. medica L. made at Aberystwyth showed, in agreement with the results of Varga, that the inhibitor $\beta$ is located at $R_{F} 0 \cdot 6-0 \cdot 8$ when chromatographed on paper with isopropanol/aqueous ammonia/water as solvent. After chromatography of the eluate with $n$-butanol/aqueous ammonia as solvent the inhibitor was located at $R_{F} 0 \cdot 4-0 \cdot 6$. This corresponds with the position of the inhibitor from $A$. pseudoplatanus in this solvent system. There were no reactions indicative of phenolic compounds in this region when chromatograms were sprayed with detecting reagents. Pure samples of the phenolic compounds mentioned by Varga were cochromatographed on paper in $n$-butanol/aqueous ammonia with the eluate from $R_{F} 0 \cdot 6-0.8$ of chromatograms of the ether extract from $C$. medica L. developed with isopropanol/aqueous ammonia/water. The $R_{F}$ of the phenolic compounds did not correspond with the zone $R_{F}$ 0.4-0.6 where inhibitor was detected by bioassay. Thus, the phenolic compounds mentioned by Varga are apparently not the major source of inhibition of extension-growth in coleoptile sections grown in the eluate of inhibitor $\beta$ from $C$. media L.; the correspondence in the $R_{F}$ values in two different solvent systems suggests that possibly the $\beta$-inhibitor in this species is related to the inhibitor of $A$. pseudoplatanus.

We thank Dr. J. W. Cornforth of 'Shell' Research, Ltd., for his helpful advice and collaboration on the chemical aspects of the work.

P. M. RobINSON

P. F. WAREING

T. H. THOMAS

Department of Botany,

University College of Wales, Aberystwyth.

1 Wareing, P. F., Physiol. Plant., 7, 261 (1954).

2 Hemberg, T., Physiol. Plant., 2, 37 (1949).

s Phillips, I. D. J., and Wareing, P. F., J. Exp. Bot., 10, 504 (1959).

4 Varga, M., Acta Biol. Szeged., 3, 213 (1957).

\title{
FUNCTIONAL COUPLING BETWEEN CELLS IN THE VISUAL CORTEX OF THE UNRESTRAINED CAT
}

\author{
By DR. J. S. GRIFFITH and DR. G. HORN \\ Departments of Theoretical Chemistry and Anatomy, University of Cambridge
}

$\mathrm{T}$

HE discharge of cells in the visual cortex of two conscious, dark-adapted cats was recorded ${ }^{1}$. The depth at which the first unit showing a resting discharge was encountered by the microelectrode was taken as zero, and all recordings were made from units between this point and another lying not more than $3 \mathrm{~mm}$ deeper in the line of penetration. Spikes were amplified, monitored on an oscilloscope and recorded on magnetic tape. When an experiment was completed, the material stored on the tape was displayed on a cathode ray tube. Occasionally the electrode recorded the simultaneous discharge of two or three cells (cells 1, 2 and 3). When it was possible, on the basis of differences in polarity, amplitude and/or shape for the experimenter to discriminate reliably between the two or three sets of action potentials on the oscilloscope, the discharges were filmed. Records of 8 such pairs of cells were analysed together with one record which contained the action potentials of three cells. Each length of film was carefully examined manually, and each spike was classified as belonging to cell 1, 2 or 3 and marked accordingly.

The positions of spikes on the film were then measured in the following way. A length of film was laid across a screen over which would be drawn a cursor line moving from left to right over a distance of $10 \mathrm{in.} \mathrm{Movement}$ of the cursor line was measured automatically and the distances from zero at left automatically set up on a counter. The cursor line was placed over a spike and by pressing a button the reading on the counter was punched on five level paper tape. The cursor line was moved to the next spike and the procedure repeated. Spikes were identified on the punched paper tape by a symbol according to whether they were from cell 1,2 or 3 . The upper limit of accuracy of the measuring instrument was 0.001 in., and on no occasion was the interval between successive spikes less than this. When a 10-in. length of film had been measured in this way both film and cursor were drawn to the left, the counter set to zero and a symbol indicating overflow punched on the paper tape. The distances of spikes on the succeeding $10 \mathrm{in.}$ of film were

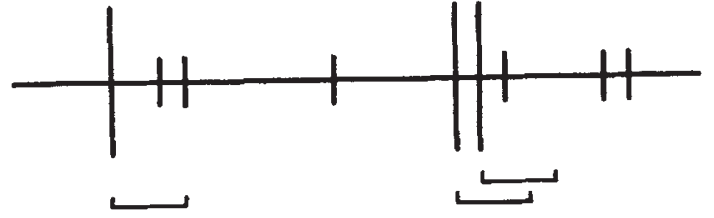

Fig. 1. Schematic diagram of a section of fllm containing spikes due to two cells. The square brackets show 5 -msec intervals following
cell 1 spikes

then measured from the new position. This procedure was repeated until the whole reel of film had been measured. The punched paper tape was printed out and compared in detail with the films to make sure the number and general distribution of spikes was correct and that the overflow symbols were correctly inserted. The paper tapes were then fed into a digital computer $(E D S A C I I)$ with a programme which gave further checks on the accuracy of the punching. Finally, the tapes were subjected on the computer to the analysis described here.

Statistical procedures. The object of the analysis was to see whether the firing of one of a pair of cells influenced the probability of discharge of the other. It seemed reasonable to suppose that this influence, if it were present, would last for a period of 10 or perhaps 100 msec. Accordingly we proceeded in the following way. Each time there was a spike due to cell 1 the number $x_{2}(0,5)$ of spikes due to cell 2 in the next $5 \mathrm{msec}$ was recorded. All these numbers were added together to give the total number $X_{2}(0,5)$ of spikes of cell 2 which occurred within 5 msec after a spike of cell 1 . In Fig. I three cell 1 spikes are shown together with their associated 5 msec intervals. The three corresponding values of $x_{2}(0,5)$ are 2,1 and 1 so that $X_{2}(0,5)=4$. It will be noticed that the coll 2 spike which occurs in both the two overlapping intervals gives two contributions to $X_{2}(0,5)$. Calculating the totals in this way is convenient because it then becomes easy to calculate the expected value of $X_{2}(0,5)$.

(Continued on page 893) 


\section{(Continued from page 876)}

In addition to finding the value $x_{2}(0,5)$, the nineteen quantities $x_{2}(5,10), x_{2}(10,15) \ldots x_{2}(95,100)$ were recorded and again totalled to give $X_{2}(5,10), X_{2}(10,15)$ $\ldots X_{2}(95,100)$. These totals refer to the number of times cell 2 spikes occur in successive 5 -msec intervals after the firing of cell 1 , up to 100 msec.

We were also interested in whether or not the firing of cell 2 influenced the subsequent firing of cell 1 ; so the same quantities were recorded with the roles of the two cells interchanged. These totals are $X_{1}(0,5), X_{1}(5,10) \ldots$ $X_{1}(95,100)$.

We now assume the cells are firing independently of each other and calculate the expected values of the $X_{1}$ $(a, a+5)$ and $X_{2}(a, a+5)$. Let there be $n_{1}$ and $n_{2}$ spikes of cell 1 and 2 respectively and the duration of the record be $T$ seconds. Further, let $m_{1}$ and $m_{2}$ be the mean rate of firing per second of each of the two cells. Then $n_{1} / T$ and $n_{2} / T$ are the sample estimates of $m_{1}$ and $m_{2}$. The probabilities of a cell firing in a randomly selected short time $\delta t$ are $m_{1} \delta t$ and $m_{2} \delta t$ for cell 1 and 2 respectively. For a longer period $\Delta t$, the expectations of the number of spikes are $m_{1} \Delta t$ and $m_{2} \Delta t$. Taking $\Delta t=5 \mathrm{msec}$, the estimate for the expected value of any $x_{2}(a, a+5)$ is $n_{2} / 200 T$ and for any $x_{1}(a, a+5)$ is $n_{1} / 200 T$.

Let us now consider, for clarity of exposition, a specific one of our totals, say, $X_{2}(0,5)$, which has in fact been analysed in detail and is described here. This value is the total number of spikes in $n_{1}$ separate 5 msec intervals, in each of which the expected number of spikes is $m_{2} / 200$. The numbers in the various intervals cannot be regarded as independent random variables because the distribution of the intervals depends on the statistical properties of the sequence of spikes of cell 1 . However, the expectation of the sum is equal to the sum of the expectations even when the numbers are not independent (see ref. 2, p. 73) and hence the expectation of $X_{2}(0,5)$ is $n_{1} m_{2} / 200$. All the $X_{2}(a, a+5)$ have the same expectation for which the sample estimate is $n_{1} m_{2} / 200=n_{1} n_{2} / 200 T$. The corresponding quantities for $X_{1}(a, a+5)$ are $n_{2} m_{1} / 200=$ $n_{1} n_{2} / 200 T$. The expected value is therefore the same for both $X_{1}$ and $X_{2}$.

Without a very detailed statistical analysis, which we hope to make later, it is not possible to give a completely rigorous test for whether an observed value for an $X_{1}$ or $X_{2}$ differs significantly from an expected one. However, it is essential to have some idea of what devia. tions may be regarded as significant and fortunately it is easy to derive an approximate test for a cell which fires fairly slowly relative to the interval considered and which occurs in a pair of cells, both of which do not have an entirely regular firing pattern.

We now write $p_{1}$ or $p_{2}$ for the probability that, respectively, cell 1 or cell 2 fires in a 5-msec interval. Clearly $p_{1} \approx n_{1} / 200 T$ and $p_{2} \approx n_{2} / 200 T$. We suppose $p_{1}$ and $p_{2}$ to be small so that $1-p_{1} \approx 1$ and $1-p_{2} \approx 1$, and we also assume that the probability of two or more firings of either cell in one interval may be neglected. Consider now $X_{2}(0,5)$. Its distribution will be given approximately by a binomial distribution with parameters $p_{2}$ and $n_{1}$ (not strictly because the different $x_{2}(0,5)$ are not entirely independent). This distribution in turn is approximated by a normal distribution with mean $n_{1} p_{2}$ and standard deviation $\sqrt{n_{1} p_{2}\left(1-p_{2}\right)}$ (see ref. 2, section 6.4). Using our sample estimate for $p_{2}$ we test the significance of the observed value of $X_{2}$ by assuming it to be a member of a normal distribution with mean $m=n_{1} n_{2}$ $200 T$ and standard deviation $\sigma=\sqrt{m}$. Owing to the symmetry of the result, the same test applies to $X_{1}$. This test should be quite reliable for our results because, with one exception, all values of $p_{1}$ and $p_{2}$ are very small.

The other parameter of cell discharge which we considered was a function of the number of spikes from one cell of a pair counted in the 100 -msec interval following the discharge of a spike in the other cell. This function was the mean, $M_{1}$, of all twenty $X_{1}$, that is, $M_{1}=\Sigma X_{1} / 20$ or the mean, $M_{2}$, of all twenty $X_{2}$, that is, $M_{2}=\Sigma X_{2} / 20$. Thus $M_{1}$ is the number of spikes from cell 1 present in the mean of 20 successive 5 -msec intervals following a spike in cell 2 and vice versa for $M_{2}$. This 5 -msec period will be referred to as the averaged 5-msec interval. In view of the analysis outlined in the preceding paragraphs it seems reasonable to compare the distribution of each of the two quantities $M_{1}$ and $M_{2}$ with a normal distribution having a mean $m=n_{1} n_{2} / 200 T$ and standard deviation $\sigma_{1}=\sqrt{(m / 20)}$.

To summarize, we have examined the question of whether the firing of one of a pair of cells (say, cell 1) influences the probability of discharge of the other (cell 2), by considering the number of spikes of cell 2 present in (i) the first 5-msec interval and (ii) the average of the twenty successive 5-msec intervals after the dis. charge of a spike from cell 1 . We have analysed these results to see whether the values so observed deviated from the expected values which were estimated.

In order to assess the statistical significance of any deviation, a parameter was calculated which was a numerical statement of the magnitude of the deviation. In the case of the data from the first 5-msec interval, the parameter was $\lambda=(X-m) / \sigma$ and in the case of the averaged 5-msec interval $\Lambda=(M-m) / \sigma_{1}$. If the value of $|\lambda|$ or $|\Lambda|$ exceeded 3 , it was considered that the deviation of observed from expected value was likely to occur by chance only rarely $(P<0.01)$ and hence reasonable to conclude that the two values came from different populations.

Experimental results. Our experimental records comprise a total of 16,770 spikes corresponding to eight recording points with two cells and one recording point with three cells. In the case of three cells, the analysis was performed separately for each of the three possible pairs.

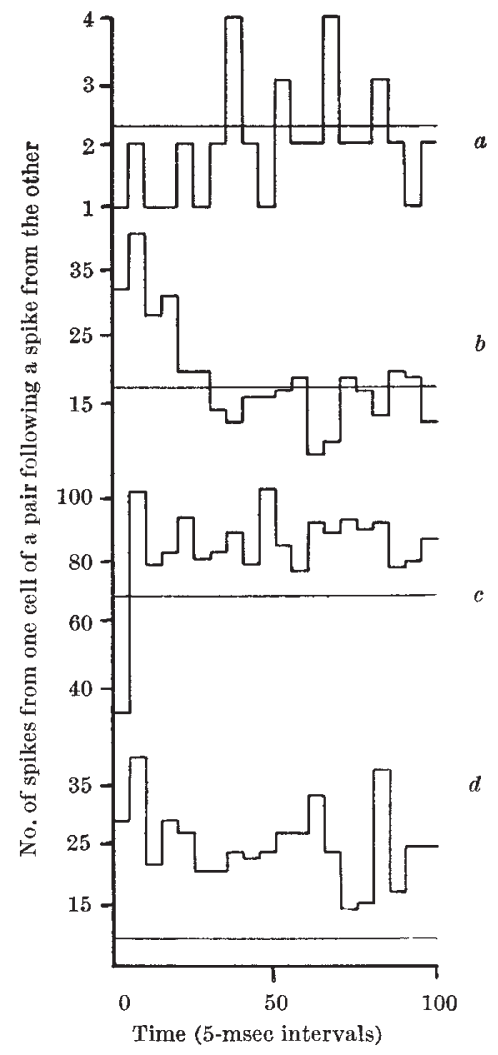

Fig. 2. Histograms showing, for selected pairs of cells, the number of spikes from one cell in successive 5 -msec intervals after the firing of the other. The expected number is drawn as a horizontal line. The in $2 ;(c)$ par 2 , pair 1 , cllow 2 following $1 ;(b)$ pair 6 , cell 1 follow 
Table 1. Propertes of the Ceilds

$n_{1}$ is the total number of spikes due to cell 1 which were counted. $m_{1}$ is the mean rate of discharge of cell 1 in spikes/sec and $p_{1}$ the probability that cell 1 will fire in any randomly selected 5 -msec interval. $n_{2}, m_{2}$ and $p_{2}$ are corresponding values for oell 2 . $T$ is the number of seconds over which the

\begin{tabular}{|c|c|c|c|c|c|c|c|c|}
\hline Cell pair & $n_{1}$ & $m_{1}$ & $p_{1}$ & $n_{2}$ & $m_{2}$ & $p_{2}$ & $T$ & Cat \\
\hline $\begin{array}{l}1 \\
2 \\
3 \\
4 \\
5 \\
6 \\
7 \\
8^{*} \\
9(12) \\
9(23) \\
9(31)\end{array}$ & $\begin{array}{r}1,149 \\
355 \\
385 \\
605 \\
641 \\
\mathbf{5 5 4} \\
1,792 \\
2,707 \\
158 \\
133 \\
439\end{array}$ & $\begin{array}{r}2 \cdot 09 \\
2 \cdot 31 \\
1 \cdot 17 \\
4 \cdot 91 \\
11 \cdot 50 \\
3 \cdot 92 \\
3 \cdot 79 \\
6 \cdot 35 \\
1 \cdot 46 \\
1 \cdot 23 \\
4 \cdot 06\end{array}$ & $\begin{array}{c}0.0104 \\
0.0115 \\
5 \cdot 87 \times 10^{-3} \\
0.0246 \\
0.0575 \\
0.0196 \\
0.0190 \\
0.0678 \\
7 \cdot 31 \times 10^{-3} \\
6.16 \times 10^{-3} \\
0.0203\end{array}$ & $\begin{array}{r}46 \\
5,811 \\
123 \\
168 \\
318 \\
853 \\
498 \\
35 \\
133 \\
439 \\
158\end{array}$ & $\begin{array}{c}0.084 \\
37.81 \\
0.38 \\
1.36 \\
5.70 \\
6.03 \\
1.05 \\
0.08 \\
1.23 \\
4.06 \\
1.46\end{array}$ & $\begin{array}{c}4 \cdot 2 \times 10^{-4} \\
0 \cdot 189 \\
1.87 \times 10^{-3} \\
6.82 \times 10^{-3} \\
0.0285 \\
0.0302 \\
5 \cdot 27 \times 10^{-3} \\
8.7 \times 10^{-4} \\
6 \cdot 16 \times 10^{-3} \\
0.0203 \\
7 \cdot 31 \times 10^{-3}\end{array}$ & $\begin{array}{r}551 \cdot 0 \\
153 \cdot 7 \\
328 \cdot 1 \\
123 \cdot 2 \\
55 \cdot 8 \\
141 \cdot 4 \\
472 \cdot 4 \\
426 \cdot 5 \\
108 \cdot 0 \\
108 \cdot 0 \\
108 \cdot 0\end{array}$ & $\begin{array}{l}35 \\
35 \\
35 \\
36 \\
36 \\
36 \\
36 \\
35 \\
36 \\
36 \\
36\end{array}$ \\
\hline
\end{tabular}

* Refers to $10 \cdot 7$-msec. intervals

Table 2. Correlation of Activities of Ceuls $X_{1}$ and $X_{2}$ are the numbers of discharges of cells 1 and 2 , respectively, in the first 5 -msec interval following discharge of the other cell. $M_{1}$ and $M_{2}$ are
the means for the first twenty 5 msec (except for cell pair 8 , for which the interval was always $10 \cdot 7$ msec). $\lambda$ and $\Lambda$ measure the deviations from the expected values, signiflcant deviations being underlined

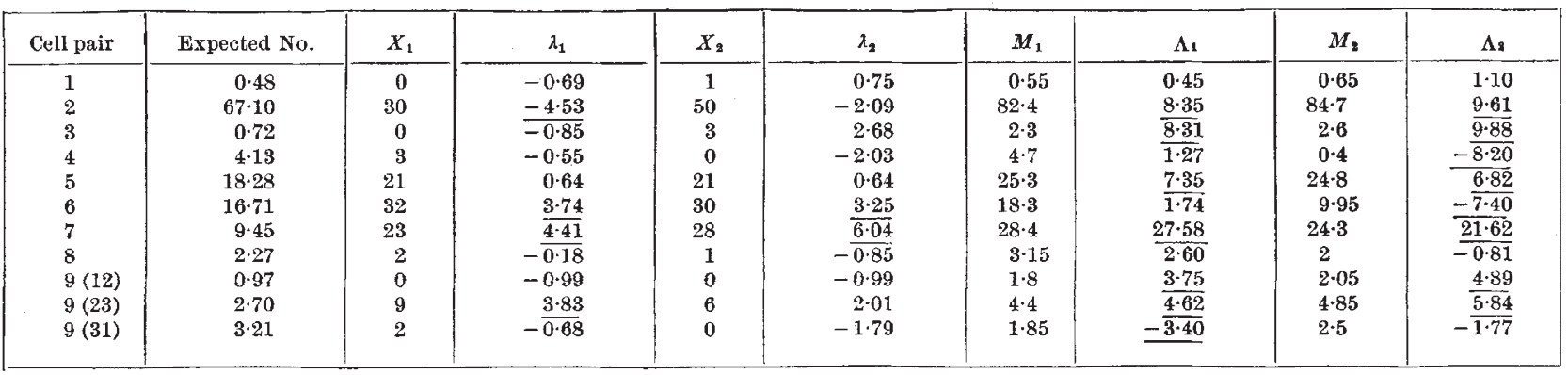

For one of the recording points with two cells the interval of time discussed in the last section was 10.7 msec, not $5 \mathrm{msec}$, owing to a different rate of recording on film. This is indicated in Table 1 .

The mean rate of discharge $\left(m_{1}\right.$ and $m_{2}$, Table 1$)$ of the nineteen cells ranged from 0.082 to $37 \cdot 8$ spikes per sec with only one cell firing at a rate exceeding $11 \cdot 5 / \mathrm{sec}$. Accordingly, the probability that a cell would fire in any randomly selected 5 msec interval $\left(p_{1}\right.$ and $p_{2}$, Table 1$)$ was, with this one exception, low.

First 5-msec interval (Table 2). In four pairs of cells the number of spikes observed from one cell in the first $5 \mathrm{msec}$ following a spike from the other, was significantly $(|\lambda|>3)$ different from the expected number. In cell pair $2, \lambda_{1}$ was a negative quantity, which means that the discharge of cell 1 was reduced to below the expected level following a spike from cell 2. The value of $\lambda_{1}$, in pair 9 (23), was positive; so the number of cell 1 spikes following a cell 2 spike was significantly greater than the expected value. In cell pair $7, \lambda_{1}$ and $\lambda_{2}$ were significant and positive. That is, the number of spikes of cell 1 following a discharge from cell 2, and the number of spikes of cell 2 following a spike from cell 1, were significantly greater than the expected value. A similar relationship existed between both cells of pair 6 .

Averaged 5-msec interval (Table 2). In two cell pairs ( 1 and 8 ) the observed number of spikes did not differ from the expected number $(|\Lambda|<3)$. That is, the discharge of a spike from one cell did not influence the probability of discharge of a spike from the other. In three cell pairs $(4,6,9(31))$ the deviation of the observed value from the expected value was significant for one of the cells of each pair. In each case, $\Lambda$ had a negative value. This parameter was both positive and significant for the two cells in each of the remaining pairs.

The magnitude and sign of $\Lambda$ for a given cell pair bore no obvious relation to the magnitude and sign of $\lambda$ for that cell pair.

The way in which the values $X(0,5), X(5,10) \ldots$ $X(95,100)$ for one cell of a pair fluctuated about the expected value was not constant from one cell to the next.
We have illustrated four fairly typical histograms of these fluctuations in Fig. 2. Each block of the histogram repre. sents the mean number of spikes of one cell in a 5-msec interval following the discharge of a spike in the other cell. The twenty intervals from this event to 100 -msec later are plotted. The horizontal line in each histogram represents the expected number of spikes. The histogram of Fig. $2 a$ is that of cell 2 following cell 1 of pair 8 . It may be seen that the observed number fluctuates irregularly about the expected value. In this case (Table 2) $\lambda_{2}$ and $\Lambda_{2}$ were both well below 3. Fig. $2 b$ is the histogram for cell 1 following a spike in cell 2 in pair 6 . After the first $20 \mathrm{msec}$, during which the number of cell spikes was increased well above the expected number, the histogram falls precipitously and fluctuates close to the expected level. In this ease $\lambda_{1},>3$, but $\left|\Lambda_{1}\right|<3$. The histogram of cell 1 following cell 2 of pair 2 is illustrated in Fig. 2c. For the first 5 msec the number of spikes is well below the expected level $\left(\lambda_{1}=-4.53\right)$, and thereafter remains above it, not returning to the expected level throughout the 100 msec analysed $\left(\Lambda_{1}=+8 \cdot 35\right)$. Cell pair 7 also shows a rather curious pattern of behaviour. The data for cell 2 following a spike in cell 1 are plotted in Fig. $2 d$. Following the cell 1 spikes the number of spikes of cell 2 is increased for each of the twenty 5 -insec periods, at no time returning to the expected value. $W_{\theta}$ anticipate that if we had continued our analysis for a long enough period (for example, $10 \mathrm{sec}$ ) following the spike in cell 1 the observed number would fall to the expected number, for it must do so ultimately.

Discussion. With the exception of two cell pairs (1 and 8) the firing of one cell of a pair was influenced by the presence of a spike in the other. Sometimes (6 cell pairs) the influence operated both ways. These effects were demonstrated by at least one of the two analyses undertaken. The influence lasted a variable length of time. In some instances (Figs. $2 c$ and $d$ ) a discharge from one cell altered the probability of discharge in the other for 100 msec. This effect was seen in a pair of cells which fired relatively slowly (pair 7, Fig. 2d) and in a pair (pair 2, Fig. 2c), one cell of which fired relatively rapidly (38 
spikes/sec). The effect thus seems to be independent of the rate of discharge of the interacting units.

When the observed number of spikes differed significantly from the expected number in both cells of a pair the deviation was positive. When the observed number differed significantly from the expected number for only one cell of a pair, the deviation usually ( 4 out of 5 occasions) was negative. The physiological interpretation of these findings is almost entirely a matter of speculation. Where both cells influence each other they may do so by mutual facilitatory interaction. It is possible, however, that the discharge of the two cells is governed by a source common to both. If the activity of this source periodically waxed and waned, it could certainly produce the coupling effects which have been described. There is no suggestion whatever that the supposed source is common to all pairs of cells showing mutual interaction.

This hypothesis is less plausible for those pairs in which the firing of only one of the cells was influenced by the other. In such pairs it is possible that one cell tends (usually) to inhibit the other, though we might have expected the effect to have been reciprocal (see, for example, ref. 3).

Finally, it is of considerable interest that each of the two cell pairs ( 1 and 8 ) which showed no evidence of interaction contained a regularly firing cell ${ }^{4}$ which discharged one spike every 12 sec approximately.

We thank the Radio Astronomy Group at the Cavendish Laboratory for their advice and assistance, the staff of the Mathematics Laboratory, especially Dr. David Wheeler, for their help and advice with programming, and the Medical Research Council for financial assistance.

1 Horn, G., J. Physiol., 158, 9P (1961).

${ }^{2}$ Cramér, H., The Elements of Probability Theory (John Wiley and Sons, New York, 1959).

3 Hartline, H. K., Wagner, H. G., and Ratliff, F., J. Gen. Physiol., 39, 651 (1956).

4 Horn, G., Nature, 194, 1084 (1962).

\title{
A RAPID METHOD FOR EXAMINING STEAM-CORROSION AND STEAM-CORROSION INHIBITION
}

\author{
By LARS G. HOLMLUND
}

\author{
Royal School of Dentistry, Umeå, Sweden
}

\begin{abstract}
YORROSION investigations have been carried out in 1 the past both as practical tests and laboratory investigations. In the former so-called 'field tests', long corrosion periods were used and the attack was usually measured by registering the change in weight of the test sample and also by comparison with standard samples the corrosion of which had been graduated according to certain scales of measurement. The corrosion specimens varied in size and shape depending on the test involved. In field tests, it is desirable to use flat specimens with large surface areas. The laboratory investigations, on the other hand, were performed using small test samples of different shape. When the corrosion in these latter tests was not measured electrochemically, the test periods were often long, the length depending on the use to which the laboratory-type corrosion tests were to be put ${ }^{1-3}$. Other workers ${ }^{4-6}$ have investigated a number of volatile amines in steam-heating systems and obtained evidence of considerable reductions in corrosion. Analogous investigations have, however, not been performed with regard to instrument sterilization in autoclaves.
\end{abstract}

The aim of the present investigation has been to investigate the corrosion of instruments during their sterilization in autoclaves and the possibilities of inhibiting this effect. For practical reasons, it has been necessary to carry out the investigations on small specimen rods. Moreover, during instrument sterilization, the corrosion times were relatively short. In order to examine more thoroughly the degree of corrosion and the effect of the corrosion inhibitor, model experiments were performed in glass apparatus in which the test samples were subjected to the influence of the same corrosion medium as in the autoclave (that is, steam and steam condensate) or to this medium together with a certain amount of inhibitor, that is, volatile amines. The temperature of the steam and steam condensate was $98^{\circ}-99^{\circ} \mathrm{C}$ and the pressure atmospheric, and in this respect the experimental conditions differed from those in autoclave sterilization where both the pressure and temperature are higher.

De-ionized water or de-ionized water containing $0 \cdot 1$ per cent cyclohexylamine was poured into a number of 'Pyrex' vessels equipped with ground-glass countercurrent coolers (Fig. 1). In each apparatus, a test sample was placed above the liquid surface on a 'Pyrex' glass support. The apparatus was then immersed in a constant-tempera- ture water bath set at $99^{\circ} \mathrm{C}$. Losses of liquid from the vessels were prevented by the counter-current coolers. Immersion lasted $120 \mathrm{~min}$.

In this way the test sample was subjected to the infuence of both steam and condensate. The specimen rods, which were cylindrical (length $45 \mathrm{~mm}$ and diameter $5 \mathrm{~mm}$ ), were made from the basic materials used for different dental instruments. In this report, results are given for one type of material (SANDVIK 21T10P. Analysis (per cent): $\mathrm{C}=1 \cdot 25 ; \mathrm{Si}=0 \cdot 15 ; \mathrm{Mn}=0 \cdot 3 ; \mathrm{Cu}=$ $0.25 ; \mathrm{W}=1 \cdot 7 ; \mathrm{V}=0.1 ; \mathrm{Pb}=0.2)$ used in dental burrs.

The specimen rods, which had been stored by rubbing with oil, were selected at random, washed with ether and rinsed in de-ionized water as cleansing liquid. Then they were 'set to zero' by means of ultrasonic cleaning immediately before the start of the tests (Narda ultrasonic apparatus: Ser. 600).

Five test samples were cleaned at a time. Each sample was cleaned by itself in a separate 'Pyrex' test-tube using $5 \mathrm{ml}$. de-ionized water. The five test-tubes were fixod in special positions in a support in the ultrasonic apparatus. The ultrasonic cleaning has been carefully standardized (time, water temperature, depth below water surface,

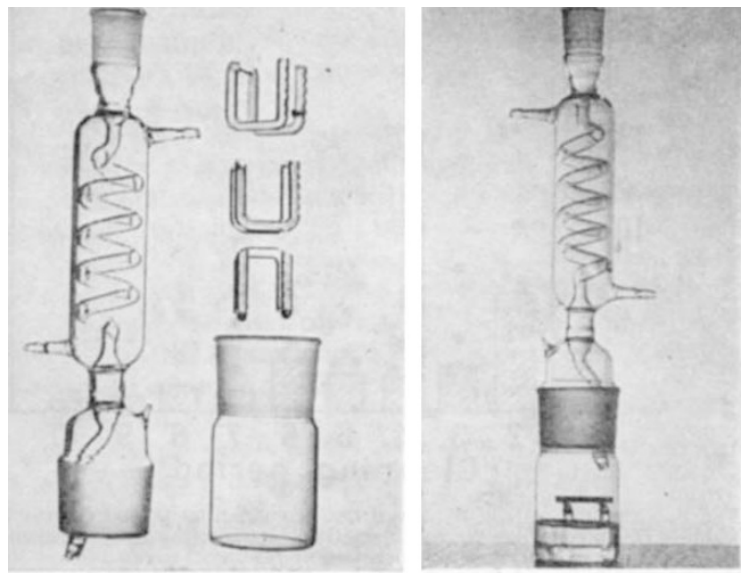

Fig. 1. 'Pyrex' corrosion apparatus. Individual parts on the left and assembled on the right 